\title{
Extubation in the operating room results in fewer composite mechanical ventilation- related adverse outcomes in patients after liver transplantation: a retrospective cohort study
}

\author{
Yan Xu' ${ }^{1}$, Yiding Zuo ${ }^{1}$, Li Zhou' ${ }^{1}$ Xuechao Hao ${ }^{1}$, Xiao Xiao ${ }^{1}$, Mao Ye ${ }^{1}$, Lulong Bo ${ }^{2}$, Chunling Jiang ${ }^{1 *}$ and \\ Jiayin Yang ${ }^{3^{*}}$
}

\begin{abstract}
Background: To investigate the effect of extubation in the operating room (OR) on mechanical ventilation-related adverse outcomes in patients who undergo liver transplantation.

Methods: Patients who underwent liver transplantation between January 2016 and December 2019 were included. According to the timing of extubation, patients were divided into OR extubation group and intensive care unit (ICU) extubation group. The propensity score was used to match OR extubation group and ICU extubation group at a 1:2 ratio by demographical and clinical covariates. The primary outcome was a composite of mechanical ventilationrelated adverse outcomes, including 30-day all-cause mortality, in-hospital acute kidney injury (stage 2 or 3), and in-hospital moderate to severe pulmonary complications. Secondary outcomes included in-hospital moderate to severe infectious complications, unplanned reintubation rates, ICU and postoperative hospital lengths of stay, and total hospital cost.

Results: A total of 438 patients were enrolled. After propensity score matching, 94 patients were in OR extubation group and 148 patients were in ICU extubation group. Incidence of the composite mechanical ventilation-related adverse outcomes was significantly lower in OR extubation group than ICU extubation group, even after adjusting for confounding factors (19.1\% vs. 31.8\%; Odds Ratio, 0.509; 95\% Confidence Index [CI], 0.274-0.946; $P=0.031$ ). The duration of ICU stay was much shorter in OR extubation group than ICU extubation group (median 4, Interquartile range $[\mathrm{IQR}](3 \sim 6)$ vs. median 6, IQR (4 8); P<0.001). Meanwhile, extubation in the OR led to a significant reduction of total hospital cost compared with extubation in the ICU (median 3.9, IQR (3.5 4.6) 10000 US dollars vs. median 4.1, IQR (3.8 5.1) 10000 US dollars; $P=0.021)$. However, there were no statistically significant differences in moderate to severe infectious complications, unplanned reintubation rates, and the length of postoperative hospital stay between groups.
\end{abstract}

\footnotetext{
*Correspondence: jiangchunling@scu.edu.cn; doctoryjy@scu.edu.cn

${ }^{1}$ Department of Anesthesiology, West China Hospital, Sichuan University

\& The Research Units of West China (2018RU012), Chinese Academy of Medical Sciences, Chengdu 610041, China

${ }^{3}$ Department of Liver Surgery and Liver Transplantation Center, West China Hospital, Sichuan University \& The Research Units of West China (2018RU012), Chinese Academy of Medical Sciences, Chengdu 610041 China

Full list of author information is available at the end of the article
} permits use, sharing, adaptation, distribution and reproduction in any medium or format, as long as you give appropriate credit to the original author(s) and the source, provide a link to the Creative Commons licence, and indicate if changes were made. The images or other third party material in this article are included in the article's Creative Commons licence, unless indicated otherwise in a credit line to the material. If material is not included in the article's Creative Commons licence and your intended use is not permitted by statutory regulation or exceeds the permitted use, you will need to obtain permission directly from the copyright holder. To view a copy of this licence, visit http://creativecommons.org/licenses/by/4.0/. The Creative Commons Public Domain Dedication waiver (http://creativecommons.org/publicdomain/zero/1.0/) applies to the data made available in this article, unless otherwise stated in a credit line to the data. 
Conclusions: Among patients who underwent liver transplantation, extubation in the OR compared with extubation in the ICU, significantly reduced the primary composite outcome of 30-day all-cause mortality, in-hospital acute kidney injury (stage 2 or 3), or in-hospital moderate to severe pulmonary complications.

Trial registration: The trial was registered at www.clinicaltrials.gov with registration number NCT04261816. Retrospectively registered on 1st February 2020.

Keywords: liver transplantation, extubation in the operating room, adverse outcomes, propensity score matching

\section{Introduction}

Liver transplantation has been established as the most effective treatment for acute and chronic end-stage liver diseases [1]. Delaying extubation to maintain postoperative ventilatory support is the routine strategy for these patients [2-4]. Previous studies have suggested that postoperative mechanical ventilation with sedation may decrease surgical stress response and may improve hemodynamic stability, thereby facilitating early recovery in patients after liver transplantation [5]. Nonetheless, mechanical ventilation can provide a direct passageway for pathogens into the lungs, and reduce the mucosal immune barrier function, which may subsequently increase the risk of postoperative pulmonary complications [6, 7]. Moreover, increasing evidence showed that mechanical ventilation, through reducing cardiac output and increasing central venous pressure and diminishing renal blood flow [8], is associated with a threefold increase in the risk of acute kidney injury (AKI) [9]. Additionally, postoperative mechanical ventilation also increases medical care cost $[10,11]$ and postoperative mortality $[12,13]$.

Over the years, many studies have reported on early extubation after liver transplantation, mainly about investigating the association between extubation in the OR and postoperative pulmonary complications [14-16]. Yet, the relationship between extubation in the OR and other adverse outcomes, e.g., 30-day all-cause mortality and in-hospital AKI, which is strongly associated with mechanical ventilation [9], remains undefined. Thus, we performed this retrospective cohort study with propensity score matching to investigate the effect of extubation in the OR after liver transplantation on a composite of mechanical ventilation-related adverse outcomes, which consists of 30-day all-cause mortality, in-hospital AKI (stage 2 or 3 ), and in-hospital moderate to severe pulmonary complications.

\section{Methods}

\section{Ethical considerations}

The medical ethics committee of the Institutional Review Board of West China Hospital, Sichuan University, reviewed and approved this retrospective data-only study with a waiver of informed consent (No. 2020-014), and it was performed in accordance with Strengthening the Reporting of Observational Studies in Epidemiology (STROBE) [17] criteria (see Additional file 1: Table S1)

\section{Patients}

This retrospective cohort study involved patients from West China Hospital, Sichuan University. The study period was from January 1, 2016, to December 31, 2019, with the following inclusion criteria: (1) age $\geq 18$ years; (2) those who underwent liver transplantation. Patients were excluded if they met any of the following criteria: (1) re-transplantation; (2) multi-visceral transplantation; (3) intraoperative death; (4) severe encephalopathy (West Haven criteria III or IV); (5) those who were already intubated before liver transplantation; (6) incomplete clinical data.

Patients were divided into two groups based on the timing of their extubation: those who were extubated in the OR were grouped into the OR extubation group, and those who were extubated in the ICU were grouped into the ICU extubation group.

\section{Anesthesia Procedure}

All patients received an orthotopic liver transplantation (piggy-back or classic) procedure under general anesthesia. Surgery and anesthesia were consistently provided by transplant surgeons and anesthesiologists with at least 5 years' experience in liver transplantation, respectively.

In all cases, balanced anesthesia with inhalational agents, opioids, and muscle relaxants were performed under routine monitoring. A standardized ventilation setting protocol of tidal volume $(6$ to $8 \mathrm{~mL} / \mathrm{kg})$ and respiratory rates (10 to 16 breaths/min) were given to maintain end-tidal carbon dioxide pressures $\left(\mathrm{ETCO}_{2}\right)$ of 35 to $45 \mathrm{~mm} \mathrm{Hg}$. Intraoperative fluid, blood product transfusion, and vasopressors were managed based on the anesthesiologist's clinical judgment utilizing intraoperative thermoelectrometry, arterial blood gases, and coagulation test results.

The decision to extubate the trachea was made by the individual attending anesthesiologist in consultation with surgeons towards the end of surgery. The criteria for extubation were following: patient being awake, obedience to verbal commands, current volume above 6 
$\mathrm{mL} / \mathrm{kg}$, breath rate between 10 to 18 per minute, $\mathrm{E}_{\mathrm{T}} \mathrm{CO}_{2}$ between 35 to $45 \mathrm{~mm} \mathrm{Hg}$, and $\mathrm{O}_{2}$ saturation above $95 \%$ (with $\mathrm{FiO}_{2} \leq 40 \%$ ), hemodynamic stability, normothermia, a complete reversal of neuromuscular blockade and adequate hemostasis in the surgical field. After extubation, the patient was transferred to the ICU for further care.

Patients without plans for extubation in the OR or who failed to fulfill the criteria for OR extubation were transferred to the ICU for mechanical ventilation support. In the ICU, weaning from mechanical ventilation was left to the discretion of the ICU attending physician.

\section{Definitions of outcomes}

The primary outcome was a composite of mechanical ventilation-related adverse outcomes, which consisted of any of the following: 30-day all-cause mortality, in-hospital AKI, or in-hospital moderate to severe pulmonary complications. AKI (stage 2 or 3 ) was defined according to Kidneys Disease improving Global Outcomes criteria [18] (see Additional file 2:Table S2). The pulmonary complications were defined as the first occurrence of pulmonary infection, respiratory failure, and pleural effusion if these occurred between ICU admission and hospital discharge. The pulmonary complication was defined and graded as mild, moderate, or severe, as described by the European Perioperative Clinical Outcomes definitions [19] (see Additional file 3:Table S3).

The secondary outcomes included in-hospital moderate to severe infectious complications, unplanned reintubation rates, ICU and postoperative hospital lengths of stay, and total hospital cost. The infectious complications included any of the followings: newonset surgical site infection (superficial/deep, organ/ space), pulmonary infection, or bloodstream infection. The infectious complications were defined and graded as mild, moderate, or severe, as described by the European Perioperative Clinical Outcomes definitions [19] (see Additional file 3:Table S3). The total hospital cost included preoperative imaging and laboratory examination, drugs and intraoperative surgery-related and anesthesia-related, and treatment in the ICU and surgical complications after surgery.

\section{Data Collection}

Specially trained research personnel collected all data from the electronic health record system. Preoperative data included age, sex, body-mass-index (BMI), smoking history, drinking history, American Society of Anesthesiologists (ASA) classification, comorbidities (including cardiovascular disease, respiratory disease, stroke, renal dysfunction, and diabetes), etiology of end-stage liver diseases, model for end-stage liver disease (MELD) score, preoperative artificial liver support and basic oxygen saturation $\left(\mathrm{SpO}_{2}\right)$. The MELD score was calculated using the following formula: $\left[0.957 \times \log _{e}\right.$ (creatinine $\left.m g / d l\right)+$ $0.378 \times \log _{e}($ bilirubin $\left.m g / d l)+1.120 \times \log _{e}(I N R)+0.643\right]$ $x$ 10. In addition, preoperative laboratory data, such as hemoglobin, white blood cell (WBC), platelet count, prothrombin time (PT), activated partial thromboplastin time (APTT), international normalized ratio (INR), alanine transaminase, total bilirubin, and albumin were also collected. The intraoperative findings included duration of surgery, urine output, the volume of packed red blood cells (PRBC) transfused, the maximal dose of vasopressors, and the maximal lactate level. The postoperative variables included the postoperative complications that occurred between ICU admission and hospital discharge, tracheal extubation time, unplanned reintubation rates, length of ICU and postoperative hospital stay, total hospital cost, and death at 30 days. The complications were examined by 2 anesthesiologists, documented in the electronic health record system and adjudicated by anesthesiologists trained in outcome definitions and unaware of the patients' groupings.

\section{Data analysis}

Categorical variables, which were presented in percentages, were analyzed using the $\chi^{2}$ test or Fisher's exact test. Continuous variables with normal distribution were reported as mean \pm standard deviation (SD) and nonnormally distributed variables as the median and interquartile range (IQR), were compared using independent Student's t-test or Mann-Whitney U test.

We used propensity score matching to reduce potential bias and confounding factors. We took all reported factors that were considered clinically important as covariates in the model to further reduce potential confounding. Final covariates included age, sex, BMI, smoking history, drinking history, ASA classification, comorbidities (including cardiovascular disease, respiratory disease, stroke, renal dysfunction, and diabetes), etiology of end-stage liver diseases, MELD score, preoperative artificial liver support, basic $\mathrm{SpO}_{2}$, preoperative laboratory data (including hemoglobin, WBC, platelet count, PT, APTT, INR, alanine transaminase, total bilirubin, and albumin) and intraoperative findings (including duration of surgery, urine output, the volume of PRBC transfused, the maximal dose of vasopressors and the maximal lactate level). A logistic regression model was used to calculate propensity scores predicting the probability of receiving OR extubation. Matching was performed with the use of a 1:2 matching protocol without replacement (greedy nearest neighbor method), with a caliper width equal to 0.2 of the standard deviation of the logit of the propensity score [20]. We used 
the standardized difference to assess balance at baseline in both groups [21]. The standardized differences of less than $10 \%$ for a given covariate indicated a relatively small imbalance [21].

Association between extubation in the $\mathrm{OR}$ and the occurrence of composite mechanical ventilation-related adverse outcomes was assessed by logistic regression analysis. Factors with $\mathrm{P}<0.10$ in univariable analyses or those considered clinically important were included in a multivariable logistic regression model to determine the risk-adjusted association between OR extubation and the risk of composite adverse outcomes development using a backward procedure. The multivariable logistic regression analysis was used to evaluate the unmatched data and matched data. In addition, we also assessed the association between baseline variables and the selection of OR extubation in all patients with logistic regression models. Factors that were considered clinically important or significant $(P<0.1)$ in univariate analysis were included in a multivariable logistic regression model to determine the independent factors that affected OR extubation by using a Enter procedure.

All reported $P$-values were two-sided, and a $P$-value $<0.05$ was considered statistically significant. Data were analyzed using SPSS $^{\circledR}$ Statistics for Windows ${ }^{\circledR}$ version 22.0 (IBM).

\section{Results}

\section{Patient Population}

Among 450 patients who were assessed for eligibility between January 2016 and December 2019; 12 patients were excluded from the study for the following reasons: re-transplantation (1 case), severe encephalopathy (3 cases), already intubated before liver transplantation (4 cases), and loss of data (4 cases) (Fig. 1). Consequently, 438 patients were included in the study (Fig. 1). After propensity score matching, 94 patients extubated in the OR were matched to 148 patients extubated in the ICU.

\section{Extubation Status}

In the entire cohort, $112(25.6 \%)$ patients were extubated in the OR, $326(74.4 \%)$ patients received ICU extubation. The median time of extubation was $12 \mathrm{~h}$ following surgery (Table 1).

In the matched cohort, 94 (38.8\%) patients received OR extubation, $148(61.2 \%)$ patients were extubated in the ICU. The median time of extubation was $11 \mathrm{~h}$ following surgery (Table 1)

\section{Baseline Characteristics}

The baseline characteristics are presented in Table 2 . Before propensity-score matching, the mean age of patients was 49 years $(S D, 10)$ in the OR extubation group, and 51 years $(\mathrm{SD}, 10)$ in the ICU extubation group $(P=0.169)$. Males made up $84.8 \%$ of the OR extubation group and $74.8 \%$ of the ICU extubation group $(P=0.029)$. There were differences in some baseline variables between the two groups. After propensity score matching, almost all baseline variables were well balanced amongst the groups (Table 2). The standardized differences were $<10.0 \%$ for most variables. Patients in the OR extubation group were younger ( $49 \pm 10 v s$. $51 \pm 10$, Standardized Difference $11.8 \%$ ) and had slightly higher preoperative albumin levels ( $38 \pm 6$ vs. $37 \pm 7$, Standardized

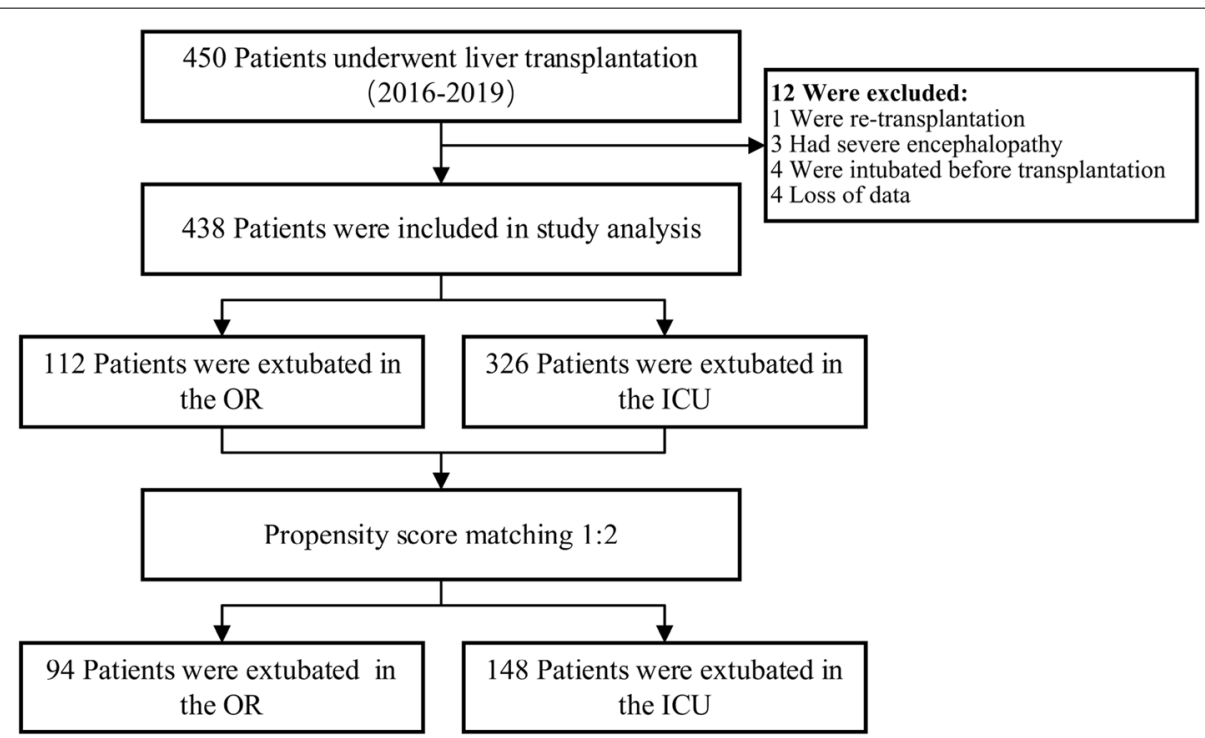

Fig. 1 Study Flow-Chart. (OR, operating room; ICU, intensive care unit) 
Table 1 Patient selections

\begin{tabular}{|c|c|c|c|c|c|c|}
\hline \multirow[t]{2}{*}{ Group } & \multicolumn{3}{|c|}{ Before matching } & \multicolumn{3}{|c|}{ After matching } \\
\hline & $\mathrm{n}$ & Percent & $\begin{array}{l}\text { Median time and range } \\
\text { of extubation }\end{array}$ & $\mathrm{n}$ & Percent & $\begin{array}{l}\text { Median time } \\
\text { and range of } \\
\text { extubation }\end{array}$ \\
\hline OR extubation group & 112 & 25.6 & / & 94 & 38.8 & / \\
\hline ICU extubation group & 326 & 74.4 & $12(8 \sim 19)$ & 148 & 61.2 & $11(8 \sim 18) h$ \\
\hline Extubation in ICU (within 24h) & 273 & 83.7 & $11(8 \sim 14)$ & 133 & 89.9 & $11(7-14) h$ \\
\hline Extubation in ICU (> 24h) & 53 & 16.3 & $39(32 \sim 54)$ & 15 & 10.1 & $33(30-48) h$ \\
\hline
\end{tabular}

Data are presented as median (interquartile range), or a number of patients (percentage), respectively

OR Operating room, ICU Intensive care unit

Difference 10.8\%) than the ICU extubation group. The estimated propensity scores before and after matching are shown in Histograms (see Additional file 4: Fig. S1).

\section{Primary Outcome}

The composite mechanical ventilation-related adverse outcomes were identified in $26.8 \%(65 / 242)$ of the matched patients. Noteworthily, patients who were extubated in the OR had a lower incidence of the composite mechanical ventilation-related adverse outcomes (19.1\%) compared to patients extubated in the ICU (31.8\%), and were associated with a significantly decreased risk of composite mechanical ventilation-related adverse outcomes (Odds Ratio, 0.509; 95\% CI, $0.274 \sim 0.946$; $P=0.031$, Table 3). Unfortunately, when analyzing each individual event that made up the composite outcome, no significant differences were found between OR extubation and ICU extubation groups in AKI (stage 2 or 3) $(P=0.181)$, pulmonary complications $(P=0.306)$, and 30 -day all-cause mortality $(P=0.081)$, as shown in Table S4 (see Additional file 5).

\section{Secondary outcomes}

After matching, the ICU length of stay decreased from the median ( $1^{\text {st }}$ quartile to $3^{\text {rd }}$ quartile) 6 ( 4 to 8 ) days in the ICU extubation group to 4 ( 3 to 6 ) days in OR extubation group $(P<0.001)$ (Table 3$)$. Moreover, extubation in the OR led to a significant reduction of total hospital cost compared with extubation in the ICU (median 3.9, IQR (3.5 4.6) 10000 US dollars vs. median 4.1, IQR (3.8 5.1) 10000 US dollars; $P=0.021)$. In addition, there were no significant differences in moderate to severe infectious complications (9.6\% vs 15.5\%; Odds Ratio, 0.575; 95\% CI, 0.254 1.304; $P=0.182$ ), postoperative hospital length of stay (median 12, IQR $(10 \sim 17)$ vs median 13, IQR (10 21); $P=0.063$ ) between OR extubation group and ICU extubation group (Table 3 ).

The incidence of reintubation in OR extubation group was not increased when compared to that in the
ICU group ( $P=0.081$ ) (Table 3$)$. A total of 26 patients (10.7\%) required tracheal reintubation during hospitalization. The causes of reintubation in the OR extubation group were respiratory distress $(n=1$ patient), reoperation $(n=4$ patients) and cardiovascular instability $(n=$ 1 patient) (see Additional file 6: Table S5); 20 patients in the ICU extubation group were reintubated due to altered mental status ( $n=1$ patient), respiratory distress ( $n=5$ patients), reoperation ( $n=11$ patients) and cardiovascular instability ( $n=3$ patients) (see Additional file 6: Table S5).

\section{Association between extubation in the OR and the composite mechanical ventilation-related adverse outcomes}

To preserve the stability of the multivariable model, 9 variables that were identified by univariable analysis $(P<0.10)$ or considered clinically important were included in a multivariable logistic regression model. After correction with confounding factors, extubation in the OR was associated with a significantly decreased risk of the composite mechanical ventilation-related adverse outcomes (Odds Ratio, 0.518; 95\%CI, 0.290 0.924; $P=0.026$ ) (Table 4). In matched patients, after correction with confounding factors, extubation in the OR remained associated with a significant reduction of the composite mechanical ventilation-related adverse outcomes (Odds Ratio, $0.514 ; 95 \%$ CI, $0.270 \sim 0.981 ; P=0.044$ ) (Table 4).

\section{Factors associated with OR extubation}

In the multivariate logistic regression analysis, 4 parameters were selected as independent predictors of OR extubation after liver transplantation (Table 5): smoking history (Odds Ratio, 0.602; 95\%CI, $0.363 \sim 0.996$;

$P=0.048$ ), duration of surgery (Odds Ratio, 0.772; 95\%CI, $0.655-0.911 ; P=0.002)$, perioperative $\mathrm{PRBC}$ transfusion units (Odds Ratio, 0.886; 95\%CI, 0.844-0.929; $P<0.001$ ) and perioperative maximal dose of vasopressors (Odds Ratio, 0.050; 95\% CI, 0.004-0.616; $P=0.019$ ). Patients 
Table 2 Baseline Characteristics Before and After Propensity Score Matching

\begin{tabular}{|c|c|c|c|c|c|c|c|c|}
\hline \multirow[t]{3}{*}{ Baseline } & \multicolumn{2}{|l|}{ Original Cohort } & \multirow[t]{3}{*}{$P$-Value } & \multirow{3}{*}{$\begin{array}{l}\text { |Standardized } \\
\text { Difference(\%)| }\end{array}$} & \multicolumn{2}{|c|}{ Propensity-matched Cohort } & \multirow[t]{3}{*}{$P$-Value } & \multirow{3}{*}{$\begin{array}{l}\text { |Standardized } \\
\text { Difference(\%) }\end{array}$} \\
\hline & OR Extubation & ICU Extubation & & & OR Extubation & ICU Extubation & & \\
\hline & $n=112$ & $n=326$ & & & $n=94$ & $n=148$ & & \\
\hline Age (y) & $49 \pm 10$ & $51 \pm 10$ & 0.169 & 15.1 & $49 \pm 10$ & $51 \pm 10$ & 0.260 & 11.8 \\
\hline \multicolumn{3}{|l|}{ Sex } & \multicolumn{2}{|l|}{0.029} & & & \multirow[t]{3}{*}{0.680} & \\
\hline Male & $95(84.8 \%)$ & $244(74.8 \%)$ & & 27.7 & $80(85.1 \%)$ & $123(83.1 \%)$ & & 1.5 \\
\hline Female & $17(15.2 \%)$ & $82(25.2 \%)$ & & 27.7 & $14(14.9 \%)$ & $25(16.9 \%)$ & & 1.5 \\
\hline BMI $\left(\mathrm{kg} / \mathrm{m}^{2}\right)$ & $22.9 \pm 2.8$ & $22.7 \pm 3.4$ & 0.440 & 8.9 & $22.9 \pm 2.8$ & $22.8 \pm 3.5$ & 0.755 & 2.5 \\
\hline Smoking & $39(34.8 \%)$ & $136(41.7 \%)$ & 0.199 & 14.4 & $36(38.3 \%)$ & $56(37.8 \%)$ & 0.943 & 1.1 \\
\hline Drinking & $26(23.2 \%)$ & $90(27.6 \%)$ & 0.363 & 10.4 & $24(25.5 \%)$ & $30(20.3 \%)$ & 0.338 & 10.0 \\
\hline \multicolumn{3}{|l|}{ ASA-classification } & \multicolumn{4}{|l|}{$<0.001$} & \multicolumn{2}{|l|}{0.051} \\
\hline ASA // & $5(4.5 \%)$ & $12(3.7 \%)$ & & 53.3 & $2(2.1 \%)$ & $10(6.8 \%)$ & & 1.1 \\
\hline ASA III & $82(73.2 \%)$ & 160 (49.1\%) & & 54.3 & $68(72.3 \%)$ & $86(58.1 \%)$ & & 0.0 \\
\hline ASA IV & $25(22.3 \%)$ & 153 (46.9\%) & & 58.8 & $24(25.5 \%)$ & $52(35.1 \%)$ & & 4.0 \\
\hline ASA V & 0 & $1(0.2 \%)$ & & / & 0 & 0 & / & / \\
\hline \multicolumn{9}{|l|}{ Comorbidity } \\
\hline $\begin{array}{l}\text { Cardiovascular } \\
\text { disease }\end{array}$ & 0 & $5(1.5 \%)$ & 0.422 & / & 0 & 0 & / & I \\
\hline $\begin{array}{l}\text { Respiratory } \\
\text { disease }^{\text {b }}\end{array}$ & $3(2.7 \%)$ & 14 (4.3\%) & 0.631 & 10.0 & $3(3.2 \%)$ & $4(2.7 \%)$ & $>0.999$ & 3.0 \\
\hline Stroke & 0 & 0 & / & / & / & / & / & / \\
\hline $\begin{array}{l}\text { Renal dysfunc- } \\
\text { tion }(\mathrm{e} G F R<60 \\
\left.\mathrm{ml} / \mathrm{min} / 1.73 \mathrm{~m}^{2}\right)\end{array}$ & $3(2.7 \%)$ & $23(7.1 \%)$ & 0.091 & 2.7 & $3(3.2 \%)$ & $5(3.4 \%)$ & $>0.999$ & 3.3 \\
\hline Diabetes & 10 (8.9\%) & $34(10.4 \%)$ & 0.648 & 5.2 & $8(8.5 \%)$ & $17(11.5 \%)$ & 0.458 & 5.6 \\
\hline \multicolumn{3}{|c|}{ Etiology of end-stage liver disease } & \multirow[t]{4}{*}{0.246} & & & & \multicolumn{2}{|l|}{0.947} \\
\hline Cirrhosis & $78(69.6 \%)$ & $206(63.2 \%)$ & & 14.0 & $63(67.0 \%)$ & $97(65.5 \%)$ & & 1.2 \\
\hline Liver cancer & $25(22.3 \%)$ & $75(23.0 \%)$ & & 1.6 & $23(24.5 \%)$ & $39(26.4 \%)$ & & 4.0 \\
\hline Other & $9(8.0 \%)$ & $45(13.8 \%)$ & & 21.0 & $8(8.5 \%)$ & $12(8.1 \%)$ & & 4.0 \\
\hline MELD score & $13(9 \sim 18)$ & $16(10 \sim 24)$ & 0.003 & 35.5 & $13(9 \sim 19)$ & $13(9 \sim 20)$ & 0.713 & 1.8 \\
\hline $\begin{array}{l}\text { Preoperative } \\
\text { artificial liver } \\
\text { support }\end{array}$ & $5(4.5 \%)$ & $31(9.5 \%)$ & 0.094 & 24.3 & $5(5.3 \%)$ & $8(5.4 \%)$ & 0.977 & 0.0 \\
\hline Basic $\mathrm{SpO}_{2}$ & $100(98 \sim 100)$ & $99(98 \sim 100)$ & 0.079 & 9.4 & $99(98 \sim 100)$ & $98(97 \sim 100)$ & 0.119 & 1.5 \\
\hline \multicolumn{9}{|c|}{ Preoperative laboratory data } \\
\hline $\begin{array}{l}\text { Hemoglobin } \\
(\mathrm{g} / \mathrm{L})\end{array}$ & $118 \pm 29$ & $110 \pm 27$ & 0.015 & 25.5 & $118 \pm 29$ & $118 \pm 27$ & 0.940 & 0.1 \\
\hline WBC $\left(\times 10^{9} / \mathrm{L}\right)$ & $4.0(2.9 \sim 5.6)$ & $4.4(3.1 \sim 6.5)$ & 0.212 & 18.0 & $3.9(3.0 \sim 5.6)$ & $4.2(3.0 \sim 6.1)$ & 0.737 & 0.9 \\
\hline $\begin{array}{l}\text { Platelet count } \\
\left(\times 10^{9} / \mathrm{L}\right)\end{array}$ & $72(41 \sim 115)$ & $67(43 \sim 106)$ & 0.598 & 2.5 & $73(43 \sim 120)$ & $66(43 \sim 104)$ & 0.482 & 6.7 \\
\hline $\mathrm{PT}(\mathrm{s})$ & $14.4(12.8 \sim 17.2)$ & $15.5(13.4 \sim 20.3)$ & 0.001 & 63.5 & $14.4(12.8 \sim 17.6)$ & $14.6(13.0 \sim 17.4)$ & 0.600 & 3.6 \\
\hline APTT (s) & $34.4(30.5 \sim 40.3)$ & $37.0(31.4 \sim 49.5)$ & 0.013 & 36.4 & $35.3(31.0 \sim 41.7)$ & $34.5(29.4 \sim 43.5)$ & 0.691 & 4.3 \\
\hline INR & $1.23(1.10 \sim 1.49)$ & $1.34(1.16 \sim 1.83)$ & 0.001 & 56.2 & $1.23(1.10 \sim 1.55)$ & $1.28(1.14 \sim 1.53)$ & 0.497 & 3.4 \\
\hline $\begin{array}{l}\text { Alanine } \\
\text { transaminase } \\
(\mathrm{IU} / \mathrm{L})\end{array}$ & $34.5(24.0 \sim 56.8)$ & $39.0(25.0 \sim 62.2)$ & 0.124 & 22.5 & $34.5(24.0 \sim 59.0)$ & $39.0(25.0 \sim 61.8)$ & 0.381 & 2.6 \\
\hline $\begin{array}{l}\text { Total bilirubin } \\
(\mu \mathrm{mol} / \mathrm{L})\end{array}$ & $\begin{array}{l}33.6(14.5 \sim \\
145.8)\end{array}$ & $\begin{array}{l}60.8(20.6 \sim \\
223.4)\end{array}$ & 0.009 & 20.6 & $34.5(14.3 \sim 155)$ & $\begin{array}{l}33.6(17.8 \sim \\
156.2)\end{array}$ & 0.725 & 2.9 \\
\hline Albumin $(\mathrm{g} / \mathrm{L})$ & $38 \pm 6$ & $36 \pm 7$ & $<0.001$ & 42.6 & $38 \pm 6$ & $37 \pm 7$ & 0.299 & 10.4 \\
\hline \multicolumn{9}{|c|}{ Intraoperative findings } \\
\hline $\begin{array}{l}\text { Duration of } \\
\text { surgery (h) }\end{array}$ & $6.4(6.0 \sim 7.0)$ & $7.0(6.0 \sim 9.0)$ & $<0.001$ & 61.6 & $7.0(6.0 \sim 7.3)$ & $7.0(6.0 \sim 8.0)$ & 0.629 & 3.8 \\
\hline
\end{tabular}


Table 2 (continued)

\begin{tabular}{|c|c|c|c|c|c|c|c|c|}
\hline \multirow[t]{3}{*}{ Baseline } & \multicolumn{2}{|l|}{ Original Cohort } & \multirow[t]{3}{*}{$P$-Value } & \multirow{3}{*}{$\begin{array}{l}\mid \text { Standardized } \\
\text { Difference(\%)| }\end{array}$} & \multicolumn{2}{|c|}{ Propensity-matched Cohort } & \multirow[t]{3}{*}{$P$-Value } & \multirow{3}{*}{$\begin{array}{l}\mid \text { Standardized } \\
\text { Difference(\%)| }\end{array}$} \\
\hline & OR Extubation & ICU Extubation & & & OR Extubation & ICU Extubation & & \\
\hline & $n=112$ & $n=326$ & & & $n=94$ & $n=148$ & & \\
\hline $\begin{array}{l}\text { Urine output } \\
(\mathrm{mL})\end{array}$ & $\begin{array}{l}1017(800 \sim \\
1500)\end{array}$ & $900(500 \sim 1400)$ & 0.026 & 21.3 & $\begin{array}{l}1000(800 \sim \\
1450)\end{array}$ & $\begin{array}{l}1000(600 \sim \\
1622)\end{array}$ & 0.643 & 1.6 \\
\hline $\begin{array}{l}\text { Packed red } \\
\text { blood cell trans- } \\
\text { fusion }(U)\end{array}$ & $4(0 \sim 8)$ & $9(4 \sim 14)$ & $<0.001$ & $>100$ & $5(0 \sim 9)$ & $6(3 \sim 9)$ & 0.155 & 5.9 \\
\hline $\begin{array}{l}\text { Maximal dose } \\
\text { of vasopressors } \\
(\mu \mathrm{g} / \mathrm{kg} / \mathrm{min})\end{array}$ & $0.08(0.05 \sim 0.15)$ & $0.10(0.07 \sim 0.20)$ & $<0.001$ & 45.2 & $0.10(0.05 \sim 0.15)$ & $0.10(0.05 \sim 0.15)$ & 0.298 & 7.0 \\
\hline $\begin{array}{l}\text { Maximal } \\
\text { lactate level } \\
\text { (mmol/L) }\end{array}$ & $5.6(4.4 \sim 8.6)$ & $7.5(5.0 \sim 11.2)$ & $<0.001$ & 46.6 & $6.4(4.4 \sim 9.4)$ & $6.4(4.3 \sim 9.2)$ & 0.930 & 1.0 \\
\hline \multicolumn{9}{|c|}{$\begin{array}{l}\text { Data are presented as mean } \pm S D \text {, median (interquartile range), or a number of patients (percentage) and compared by independent samples t-test, Mann-Whitney } U \\
\text { test or } x^{2} \text { test/Fisher's exact test, respectively }\end{array}$} \\
\hline \multicolumn{9}{|c|}{$\begin{array}{l}\text { OR Operating room, ICU Intensive care unit, BMI Body mass index, ASA Ameri } \\
\text { saturation, WBC White blood cell, PT Prothrombin time, APTT Activated partia }\end{array}$} \\
\hline \multicolumn{9}{|c|}{ a Including hypertension, coronary artery disease, congenital heart disease } \\
\hline \multicolumn{9}{|c|}{ b Including chronic obstructive pulmonary disease, pulmonary infection, pleural effusion } \\
\hline ding epine & orepinephrin & & & & & & & \\
\hline
\end{tabular}

Table 3 Outcomes Before and After Propensity Score Matching

\begin{tabular}{|c|c|c|c|c|c|c|c|c|}
\hline \multirow[t]{3}{*}{ Outcome } & \multicolumn{2}{|l|}{ Original Cohort } & \multirow{3}{*}{$\begin{array}{l}\text { Odds Ratio } \\
(95 \% \mathrm{Cl})\end{array}$} & \multirow[t]{3}{*}{$P$-Value } & \multicolumn{2}{|c|}{ Propensity-matched Cohort } & \multirow{3}{*}{$\begin{array}{l}\text { Odds Ratio } \\
(95 \% \mathrm{Cl})\end{array}$} & \multirow[t]{3}{*}{$P$-Value } \\
\hline & OR Extubation & ICU Extubation & & & OR Extubation & ICU Extubation & & \\
\hline & $n=112$ & $n=326$ & & & $n=94$ & $n=148$ & & \\
\hline \multicolumn{9}{|l|}{ Primary outcome } \\
\hline $\begin{array}{l}\quad \text { 30-day all- } \\
\text { cause mortality, } \\
\text { AKI (stage II or III) } \\
\text { and Moderate- } \\
\text { severe pulmonary } \\
\text { complications }\end{array}$ & $19(17.0 \%)$ & $125(38.3 \%)$ & $\begin{array}{l}0.329(0.191 \sim \\
0.565)\end{array}$ & $<0.001$ & $18(19.1 \%)$ & $47(31.8 \%)$ & $\begin{array}{l}0.509(0.274 \sim \\
0.946)\end{array}$ & 0.031 \\
\hline \multicolumn{9}{|c|}{ Secondary outcomes } \\
\hline $\begin{array}{l}\text { Moderate to } \\
\text { severe infectious } \\
\text { complications }\end{array}$ & $9(8.0 \%)$ & $65(19.9 \%)$ & $\begin{array}{l}0.351(0.169 \sim \\
0.731)\end{array}$ & 0.004 & $9(9.6 \%)$ & $23(15.5 \%)$ & $\begin{array}{l}0.575(0.254 \sim ~ \\
1.304)\end{array}$ & 0.182 \\
\hline $\begin{array}{l}\text { ICU length of } \\
\text { stay }\end{array}$ & $4(3 \sim 5)$ & $6(4 \sim 10)$ & / & $<0.001$ & $4(3 \sim 6)$ & $6(4 \sim 8)$ & / & $<0.001$ \\
\hline $\begin{array}{l}\text { Postoperative } \\
\text { hospital length } \\
\text { of stay }\end{array}$ & $12(9 \sim 17)$ & $15(11 \sim 22)$ & / & 0.001 & $12(10 \sim 17)$ & $13(10 \sim 21)$ & / & 0.063 \\
\hline $\begin{array}{l}\text { Unplanned } \\
\text { reintubation }\end{array}$ & $7(6.3 \%)$ & $53(16.3 \%)$ & $\begin{array}{l}0.343(0.151 \sim \\
0.779)\end{array}$ & 0.008 & $6(6.4 \%)$ & $20(13.5 \%)$ & $\begin{array}{l}0.436(0.168 \sim ~ \\
1.130)\end{array}$ & 0.081 \\
\hline $\begin{array}{l}\text { Total hospital } \\
\text { cost ( } 10,000 \text { US } \\
\text { dollars) }\end{array}$ & $3.9(3.5 \sim 4.5)$ & $4.4(3.9 \sim 5.4)$ & / & $<0.001$ & 3.9 (3.5 4.6) & $4.1(3.8 \sim 5.1)$ & / & 0.021 \\
\hline
\end{tabular}

Data are presented as mean $\pm S D$, median (interquartile range), or a number of patients (percentage) and compared by independent samples t-test, Mann-Whitney $\mathrm{U}$ test, or chi-square test/Fisher's exact test, respectively

$O R$ Operating room, ICU Intensive care unit, CI Confidence Index, AKI Acute kidney injury 
Table 4 Association between extubation in the OR and composite mechanical ventilation-related adverse outcomes ${ }^{a}$

\begin{tabular}{|c|c|c|c|c|c|c|}
\hline Characteristics & $\begin{array}{l}\text { All recipients } \\
\text { Univariable } P \\
\text { valve }\end{array}$ & $\begin{array}{l}\text { Multivariable } \\
\text { analysis Odds Ratio } \\
(95 \% \mathrm{Cl})\end{array}$ & $P$ valve & $\begin{array}{l}\text { Matched recipients } \\
\text { Univariable } P \text { valve }\end{array}$ & $\begin{array}{l}\text { Multivariable } \\
\text { analysis Odds Ratio } \\
(95 \% \mathrm{Cl})\end{array}$ & $P$ valve \\
\hline Sex & 0.029 & - & - & 0.68 & - & - \\
\hline ASA-classification (n), \% & $<0.001$ & $0.488(0.163 \sim 1.461)$ & 0.200 & 0.051 & $0.640(0.163 \sim 2.513)$ & 0.523 \\
\hline $\begin{array}{l}\text { Renal dysfunction (eGFR }<60 \mathrm{ml} / \\
\mathrm{min} / 1.73 \mathrm{~m}^{2} \text { ) }\end{array}$ & 0.091 & $2.161(0.927 \sim 5.037)$ & 0.074 & $>0.999$ & $0.680(0.139 \sim 3.316)$ & 0.633 \\
\hline MELD score & 0.003 & $0.999(0.966 \sim 1.033)$ & 0.963 & 0.713 & $1.022(0.987 \sim 1.058)$ & 0.228 \\
\hline Preoperative artificial liver support & 0.094 & $1.129(0.515 \sim 2.474)$ & 0.763 & 0.977 & $3.645(0.680 \sim 19.535)$ & 0.131 \\
\hline Basic SpO2 & 0.079 & $0.858(0.769 \sim 0.957)$ & 0.006 & 0.119 & $0.844(0.720 \sim 0.990)$ & 0.037 \\
\hline Preoperative-Hemoglobin (g/L) & 0.015 & - & - & 0.940 & - & - \\
\hline PT (s) & 0.001 & - & - & 0.600 & - & - \\
\hline APTT (s) & 0.013 & - & - & 0.691 & - & - \\
\hline INR & 0.001 & - & - & 0.497 & - & - \\
\hline Total bilirubin ( $\mu \mathrm{mol} / \mathrm{L})$ & 0.009 & - & - & 0.725 & - & - \\
\hline Albumin (g/L) & $<0.001$ & - & - & 0.299 & - & - \\
\hline Duration of surgery (h) & $<0.001$ & $1.091(0.963 \sim 1.235)$ & 0.171 & 0.629 & $1.054(0.857 \sim 1.296)$ & 0.617 \\
\hline Urine output & 0.026 & - & - & 0.643 & - & - \\
\hline Packed red blood cell transfusion (U) & $<0.001$ & $1.083(1.052 \sim 1.115)$ & $<0.001$ & 0.155 & $1.085(1.025 \sim 1.148)$ & 0.005 \\
\hline $\begin{array}{l}\text { Maximal vasopressors need }(\mu \mathrm{g} / \mathrm{kg} / \\
\min )\end{array}$ & $<0.001$ & - & - & 0.298 & - & - \\
\hline Maximal lactate (mmol/L) & $<0.001$ & $1.041(0.992 \sim 1.092$ & 0.102 & 0.930 & 1.069 (0.996 1.146) & 0.064 \\
\hline Extubation & - & $0.518(0.290 \sim 0.924)$ & 0.026 & - & $0.514(0.270 \sim 0.981)$ & 0.044 \\
\hline
\end{tabular}

ASA American Society of Anesthesiologists, MELD Model for End-Stage Liver Disease, SpO2 Oxygen saturation, PT Prothrombin time, APTT Activated partial thromboplastin time, INR International normalized ratio, Cl Confidence Index

${ }^{a}$ Composite mechanical ventilation-related adverse outcomes includes 30-day all-cause mortality, in-hospital acute kidney injury (stage 2 or 3), or in-hospital moderate to severe pulmonary complications.

with smoking history were less likely to undergo OR extubation than those without smoking history. A 1 hour increase in the duration of surgery decreased the chance for OR extubation by $22.8 \%$, transfusion of 1 unit of PRBC decreased the chance for OR extubation by $11.4 \%$, and $1 \mu \mathrm{g} / \mathrm{kg} / \mathrm{min}$ increase in the vasopressors dose decreased the chance for OR extubation by $95 \%$.

\section{Discussion}

Our analysis demonstrates that patients extubated in the OR were associated with a lower incidence of composite mechanical ventilation-related adverse outcomes, shorter ICU length of stay, and lower total hospital cost compared with ICU extubation. Unfortunately, extubation in the OR did not lead to a superior effect in moderate to severe infectious complications and postoperative hospital length of stay.

Previous studies have shown that postoperative mechanical ventilation, as the routine management strategy for most patients undergoing liver transplantation, can significantly increase the incidence of pulmonary complications [14-16]. Furthermore, mechanical ventilation has also been associated with a threefold increase in the risk of AKI [9]. Yet, it remains unclear whether these adverse outcomes can be reduced by shortening the time of mechanical ventilation through extubation in the OR. Consequently, we performed this retrospective study to explore whether extubation in the OR was associated with a lower incidence of aforementioned adverse outcomes. Considering some baseline variables that might influence the development of adverse outcomes were different between patients extubated in the OR and those extubated in the ICU, we performed propensity score matching to minimize the confounding effects due to non-randomized assignment. The factors selected for propensity score matching covered indicators previously reported in the literature $[3,22,23]$ or clinically considered to be important to the outcome, including demographic data, smoking history, drinking history, ASA classification, comorbidities, etiology of end-stage liver diseases, MELD score, preoperative artificial liver support, basic $\mathrm{SpO}_{2}$, preoperative laboratory data and intraoperative findings. Based on these factors, the baseline variables were well balanced between the two groups after matching. In matched analyses, the results showed that the composite mechanical ventilationrelated adverse outcomes occurred less frequently in the OR extubation group. Moreover, the above results were 
Table 5 Multivariate logistic regression analysis to evaluate independent factors for OR extubation

\begin{tabular}{|c|c|c|c|c|c|c|}
\hline \multirow[t]{2}{*}{ Variable } & \multicolumn{3}{|c|}{ Univariable analysis } & \multicolumn{3}{|c|}{ Multivariate logistic analysis } \\
\hline & Odds Ratio & $95 \% \mathrm{Cl}$ & $P$ valve & Odds Ratio & $95 \% \mathrm{Cl}$ & $P$ valve \\
\hline Age (y) & 0.986 & $0.965-1.006$ & 0.169 & 0.987 & $0.963-1.011$ & 0.279 \\
\hline \multicolumn{7}{|l|}{ Sex } \\
\hline Female & Ref & & & Ref & & \\
\hline Male & 1.878 & $1.058-3.333$ & 0.031 & 1.708 & $0.819-3.562$ & 0.153 \\
\hline BMI $\left(\mathrm{kg} / \mathrm{m}^{2}\right)$ & 1.024 & $0.959-1.093$ & 0.48 & 1.021 & $0.942-1.106$ & 0.614 \\
\hline \multicolumn{7}{|l|}{ Smoking } \\
\hline No & Ref & & & Ref & & \\
\hline Yes & 0.746 & $0.477-1.167$ & 0.199 & 0.602 & $0.363-0.996$ & 0.048 \\
\hline \multicolumn{7}{|l|}{ ASA-classification } \\
\hline ASA II & Ref & & & & & \\
\hline ASA III & 1.230 & $0.419-3.610$ & 0.706 & / & / & $>0.999$ \\
\hline ASA IV & 0.392 & $0.127-1.209$ & 0.103 & / & / & $>0.999$ \\
\hline ASA V & 0.000 & 0 & $>0.999$ & / & / & $>0.999$ \\
\hline \multicolumn{7}{|l|}{ Cardiovascular disease } \\
\hline No & Ref & & & & & \\
\hline Yes & 0.000 & / & $>0.999$ & / & / & $>0.999$ \\
\hline \multicolumn{7}{|l|}{ Respiratory disease } \\
\hline No & Ref & & & Ref & & \\
\hline Yes & 0.613 & $0.173-2.175$ & 0.449 & 0.838 & $0.204-3.444$ & 0.806 \\
\hline MELD score & 0.963 & $0.939-0.988$ & 0.004 & 1.017 & $0.974-1.061$ & 0.443 \\
\hline \multicolumn{7}{|l|}{ Preoperative artificial liver support } \\
\hline No & Ref & & & Ref & & \\
\hline Yes & 0.445 & $0.169-1.173$ & 0.102 & 0.502 & $0.129-1.953$ & 0.320 \\
\hline Preoperative-Hemoglobin (g/L) & 1.010 & $1.002-1.018$ & 0.015 & 0.991 & $0.980-.002$ & 0.119 \\
\hline Preoperative-WBC ( $\left.\times 10^{9} / \mathrm{L}\right)$ & 0.946 & $0.875-1.023$ & 0.162 & 0.942 & $0.850-1.043$ & 0.250 \\
\hline Preoperative-Albumin (g/L) & 1.060 & $1.025-1.095$ & 0.001 & 1.022 & $0.975-1.070$ & 0.365 \\
\hline Duration of surgery $(\mathrm{h})$ & 0.739 & $0.641-0.852$ & $<0.001$ & 0.772 & $0.655-0.911$ & 0.002 \\
\hline Urine output (mL) & 1.000 & $1.0000-1.0001$ & 0.042 & 1.000 & $1.000-1.001$ & 0.028 \\
\hline Packed red blood cell transfusion (U) & 0.867 & $0.829-0.906$ & $<0.001$ & 0.886 & $0.844-0.929$ & $<0.001$ \\
\hline Maximal vasopressors need $(\mu \mathrm{g} / \mathrm{kg} / \mathrm{min})$ & 0.017 & $0.001-0.197$ & 0.001 & 0.050 & $0.004-0.616$ & 0.019 \\
\hline
\end{tabular}

CI Confidence Index, OR Operating room, BMI Body mass index, ASA American Society of Anesthesiologists, MELD Model for End-Stage Liver Disease, WBC White blood cell

verified with logistic regression analysis. Not surprisingly, the result of logistic regression analysis was in line with matched analysis (see Additional file 7: Fig. S2). However, when analyzing each individual event that made up the composite mechanical ventilation-related adverse outcomes, no significant differences were found between the two groups, which may be due to the small sample size and not having enough power to detect clinically meaningful increases in the risk of adverse outcomes. Additionally, given the lack of available evidence, this finding is considered exploratory at this point, and future larger studies are necessary to further elucidate this aspect.

Shortening the length of ICU stay is also crucial for improving patients' prognosis since long-term ICU stay may lead to long-lasting physical, cognitive and/or mental health impairments. In this study, we found an association between extubation in the OR and shorter ICU length of stay, as well as less total hospital cost, which was consistent with previous studies [16, 24, 25]. At our center, patients undergoing liver transplantation had a relatively fixed cost of preoperative imaging and laboratory examination, drugs, surgery and anesthesia. The cost that made a difference was primarily from postoperative treatment, especially that on mechanical ventilation in the ICU and surgical complications after surgery. This was further reinforced by the finding that the cost was increased when patients were extubated in the ICU ((median 3.9, IQR (3.5 4.6) 10000 US dollars vs. median 
4.1, IQR (3.8 5.1) 10000 US dollars). Therefore, early extubation contributed to a shorter length of ICU stay after surgery and subsequently less cost. In line with these findings, a retrospective study focusing on patients undergoing liver transplantation with FastTrack protocol involving extubation in the OR also resulted in a relatively shorter ICU stay and cost without increasing pulmonary complications and reintubation rates [16]. Another study that included 64 pediatric liver transplant patients also demonstrated that OR extubation led to shorter ICU stay and earlier hospital discharge [24]. In a more broad sense, early discharge from the ICU also increases the number of beds open to other patients in need of intensive care.

In order to exclude the major differences in patient baseline characteristics, which might be responsible for the late extubation and subsequent worse postoperative outcomes and prolonged ICU length of stay and total hospital cost, we made a propensity score-matched analysis. However, there was still a small portion of extubation in the ICU mechanical time more than $24 \mathrm{~h}$ after matching. Thereafter, a comparison between patients who were extubated in the OR and in the ICU within $24 \mathrm{~h}$ was performed after excluding the patients extubated later than $24 \mathrm{~h}$ postoperatively. The results still showed a significant decrease in composite mechanical ventilationrelated adverse outcomes, ICU length of stay, and total hospital cost (see Additional file 8: Table S6).

Extubation in the OR has been proved to be safe in most liver transplantation recipients [15]. Consistent with previous studies [26], our results showed that the unplanned reintubation rates in the OR extubation group were not increased compared to patients extubated in ICU. Moreover, in most reintubation cases, the causes of early reintubation were related to urgent reoperations, whereas inadequate reawakening or respiratory failures were rarely implicated.

Logistic regression analysis of our data revealed that patient's smoking history, duration of surgery, intraoperative PRBC transfusion, and the maximal dose of vasopressors were significantly associated with OR extubation. One of the novel findings in our study is that patients without smoking history showed a significantly higher likelihood of early extubation. This may be due to the fact that patients without smoking history might have better lung function, which in turn might lead to a higher likelihood of early extubation in this population. With regard to the duration of surgery, as described by Mandell et al [27], a longer duration of surgical procedure was associated with fewer attempts at early extubation. Additionally, many studies have focused on intraoperative hemodynamic instability for predicting early extubation after liver transplantation [26], and our results also showed that intraoperative factors related to hemodynamic disturbance (i.e., the use of vasopressor, and a requirement for a large PRBC transfusion) were negatively associated with early extubation. A retrospective study by Skurzak et al. [26] suggested that patients intraoperative PRBC transfusion $<7$ units and norepinephrine $<0.05 \mu \mathrm{g} / \mathrm{kg} / \mathrm{min}$ at the end of surgery were more suitable for OR extubation. MELD score is considered a valuable tool for identifying the sickest patients. However, it remains controversial whether the MELD score can be utilized as one of the extubation criteria. Similarly to some previous studies, we found that MELD score had no impact on early extubation $[28,29]$. On the contrary, a prospective study by Biancofiore et al. [30] suggested that a MELD score of 11 points was the optimal cut-off for immediate postoperative extubation, but the sensibility and specificity were found to be low. Thus, even patients with higher MELD score may not necessarily need to be excluded from an early extubation protocol before the beginning of surgery. Based on our results and previous studies, it was shown that patients without a smoking history, short surgery time, and/or intraoperative hemodynamic stability showed a higher likelihood of operating room extubation. Therefore, for patients with above characteristics, we recommend to extubate them in the OR.

This study has several limitations. Firstly, this study was a non-randomized, observational rather than experimental study. We, therefore, performed propensity score matching to reduce potential bias and strengthen our reported effect estimates. However, it is inevitable that the use of propensity score matching will shrinking the sample size. Secondly, the lack of some data (e.g., cold ischemia time, as well as donor factors such as donor steatosis, Donation after circulatory death/Donation after Brain Death) could not be avoided because of the study's retrospective design. However, the most relevant factors that might impact the outcomes were involved in the current study. Thirdly, the judgments about tracheal extubation were subjective to some degree [25]. Thus, a standardized protocol is essential for early extubation. Fourthly, we acknowledge that the numbers of patients evaluated in this study were small. Moreover, to preserve the stability of the multivariable model, we had to limit the number of variables in the final model. We selected 22 variables for the initial univariable model. We assessed correlation among those 22 factors and eventually restricted the maximum number to 9 variables that were included in the first multivariable model. Finally, because this analysis comprised a 3-year time span of liver transplantation, some changes in perioperative procedures occurred during this time period. Therefore, further large prospective matched studies are required to determine 
whether early extubation has positive effects on adverse clinical outcomes.

\section{Conclusions}

Our data suggested that the practice of early extubation in the OR is associated with a lower incidence of composite mechanical ventilation-related adverse outcomes, shorter ICU stay, and lower total hospital cost without increasing the reintubation rates. Given the benefits of early extubation in the OR, this practice should be considered when appropriate. Meanwhile, it is important to identify liver transplantation recipients eligible for early extubation in the OR.

\section{Abbreviations}

OR: Operating Room; ICU: intensive care unit; AKI: Acute Kidney Injury; BMI: Body Mass Index; ASA: American Society of Anesthesiologists; MELD: Model for End-Stage Liver Disease; INR: International Normalized Ratio; WBC: White Blood Cell; PT: Prothrombin Time; APTT: Activated Partial Thromboplastin Time; PRBC: Packed Red Blood Cells.

\section{Supplementary Information}

The online version contains supplementary material available at https://doi. org/10.1186/s12871-021-01508-1.

Additional file 1: Table S1. STROBE Statement. Checklist of our cohort study, which demonstrates STROBE Statement Checklist of our cohort study.

Additional file 2: Table S2 Predefined Acute Kidney Injury (AKI) According Kidney Disease Improving Global Outcomes Guidelines (KIDGO).

Additional file 3: Table S3 Predefined Postoperative Complications According European Perioperative Clinical Outcome definitions.

Additional file 4: Figure S1 Histograms of the estimated propensity scores.

Additional file 5: Table S4 Postoperative complications after matching.

Additional file 6: Table S5 Unplanned-Reintubation indications amongst OR extubation and ICU extubation groups after matching.

Additional file 7: Figure S2 Association between extubation in the OR and composite mechanical ventilation-related adverse outcomes.

Additional file 8: Table S6 Outcomes between OR extubation group and ICU extubation group (within 24h) after matching.

\section{Acknowledgments}

We thank Dr. Deren Wang from the West China Hospital, Sichuan University, for instructive advice during the writing process.

\section{Authors' contributions}

$C L$, LLB, and JYY supervised the study. CLJ and YX designed the study. YDZ, $L Z, X C H, X X$, and $M Y$ recruited patients, collected baseline and perioperative data. YX, XCH, and CLJ analyzed and interpreted all the data. YX and YDZ contributed to statistical analyses. YX drafted the manuscript. CLJ, LLB, and JYY critically revised the manuscript. All authors revised the manuscript critically for important intellectual content. All authors read and approved the final manuscript.

\section{Funding}

This work was supported by the National Natural Science Foundation of China (grant number 81971806), the 1.3.5. project for the discipline of excellence, West China Hospital, Sichuan University (grant number 2018HXFH046), and the Shanghai Science and Technology Committee Rising-Star Program (grant number 19QA1408500).

\section{Availability of data and materials}

The datasets used and/or analyzed during this study are available from the corresponding author on reasonable request.

\section{Declarations}

\section{Ethics approval and consent to participate}

The medical ethics committee of the Institutional Review Board of West China Hospital, Sichuan University, reviewed and approved this retrospective study (No. 2020-014). The requirement for informed consent was waived due to the retrospective study design.

\section{Consent for publication}

Not applicable.

\section{Competing interests}

The authors declare that they have no competing interests.

\section{Author details}

${ }^{1}$ Department of Anesthesiology, West China Hospital, Sichuan University \& The Research Units of West China (2018RU012), Chinese Academy of Medical Sciences, Chengdu 610041, China. ${ }^{2}$ Department of Anesthesiology, Changhai Hospital, Naval Medical University, Shanghai 200433, China. ${ }^{3}$ Department of Liver Surgery and Liver Transplantation Center, West China Hospital, Sichuan University \& The Research Units of West China (2018RU012), Chinese Academy of Medical Sciences, Chengdu 610041, China.

Received: 14 June 2021 Accepted: 8 November 2021

Published online: 18 November 2021

\section{References}

1. Zarrinpar A, Busuttil RW. Liver transplantation: past, present and future. Nat Rev Gastro Hepat. 2013;10(7):434-40.

2. Li J, Wang C, Jiang Y, Song J, Zhang L, Chen N, et al. Immediate versus conventional postoperative tracheal extubation for enhanced recovery after liver transplantation: IPTE versus CTE for enhanced recovery after liver transplantation. Medicine (Baltimore). 2018;97(45):e13082.

3. Feltracco P, Carollo C, Barbieri S, Pettenuzzo T, Ori C. Early respiratory complications after liver transplantation. World J Gastroentero. 2013;19(48):9271-81.

4. Aniskevich S, Pai SL. Fast track anesthesia for liver transplantation: Review of the current practice. World J Hepat. 2015;7(20):2303-8.

5. Glanemann M, Langrehr J, Kaisers U, Schenk R, Muller A, Stange B, et al. Postoperative tracheal extubation after orthotopic liver transplantation. Acta Anaesth Scand. 2001;45(3):333-9.

6. Parker JC, Hernandez LA, Peevy KJ. Mechanisms of ventilator-induced lung injury. Crit Care Med. 1993;21(1):131-43.

7. Rello J, Lisboa T, Koulenti D. Respiratory infections in patients undergoing mechanical ventilation. Lancet Resp Med. 2014;2(9):764-74.

8. Legrand M, Dupuis C, Simon C, Gayat E, Mateo J, Lukaszewicz AC, et al. Association between systemic hemodynamics and septic acute kidney injury in critically III patients: a retrospective observational study. Crit Care. 2013;17(6):1-8.

9. van den Akker JP, Egal M, Groeneveld AB. Invasive mechanical ventilation as a risk factor for acute kidney injury in the critically ill: a systematic review and meta-analysis. Crit Care. 2013;17(3):R98.

10. Kahn JM, Le T, Angus DC, Cox CE, Hough CL, White DB, et al. The Epidemiology of Chronic Critical IIIness in the United States. Crit Care Med. 2015;43(2):282-7.

11. Kaier K, Heister T, Wolff J, Wolkewitz M. Mechanical ventilation and the daily cost of ICU care. BMC Health Serv Res. 2020;20(1):267.

12. McGee WT. Expectations and outcomes of prolonged mechanical ventilation. Crit Care Med. 2010;38(5):1393-4. 
13. Sun Y, Li S, Wang S, Li C, Li G, Xu J, et al. Predictors of 1-year mortality in patients on prolonged mechanical ventilation after surgery in intensive care unit: a multicenter, retrospective cohort study. BMC Anesthesiol. 2020;20(1):44

14. Steadman RH. Con: immediate extubation for liver transplantation. J Cardiothor Vasc An. 2007;21(5):756-7.

15. Mandell MS, Stoner TJ, Barnett R, Shaked A, Bellamy M, Biancofiore G, et al. A multicenter evaluation of safety of early extubation in liver transplant recipients. Liver Transplant. 2007:13(11):1557-63.

16. Acho C, Morita Y, Fernandez V, Safwan M, Galusca D, Abouljoud M, et al. Immediate Post-Operative Extubation Decreases Pulmonary Complications in Liver Transplant Patients. Transplant. 2021;105(9):2018-28.

17. von Elm E, Altman DG, Egger M, Pocock SJ, Gotzsche PC, Vandenbroucke JP. The Strengthening the Reporting of Observational Studies in Epidemiology (STROBE) statement: guidelines for reporting observational studies. Prev Med. 2007:45(4):247-51.

18. Khwaja A. KDIGO clinical practice guidelines for acute kidney injury. Nephron Clin Pract. 2012:120(4):179-84.

19. Jammer I, Wickboldt N, Sander M, Smith A, Schultz MJ, Pelosi P, et al. Standards for Definitions and Use of Outcome Measures for Clinical Effectiveness Research in perioperative medicine: european perioperative clinical outcome (epco) definitions: a statement from the esa-esicm joint taskforce on perioperative outcome measures. Eur J Anaesthesiol. 2015:32(2):88-105.

20. Austin PC. Optimal caliper widths for propensity-score matching when estimating differences in means and differences in proportions in observational studies. Pharm Stat. 2011:10(2):150-61.

21. Normand ST, Landrum MB, Guadagnoli E, Ayanian JZ, Ryan TJ, Cleary PD, et al. Validating recommendations for coronary angiography following acute myocardial infarction in the elderly: a matched analysis using propensity scores. J Clin Epidemiol. 2001:54(4):387-98.

22. Kim JM, Kim DG, Kim J, Lee K, Lee KW, Ryu JH, et al. Outcomes after liver transplantation in Korea: Incidence and risk factors from Korean transplantation registry. Clin Mol Hepatol. 2021;27(3):451-62.
23. Parikh A, Washburn KW, Matsuoka L, Pandit U, Kim JE, Almeda J, et al. A multicenter study of 30 days complications after deceased donor liver transplantation in the model for end-stage liver disease score era. Liver Transpl. 2015;21(9):1160-8.

24. Gurnaney HG, Cook-Sather SD, Shaked A, Olthoff KM, Rand EB, Lingappan $\mathrm{AM}$, et al. Extubation in the operating room after pediatric liver transplant: A retrospective cohort study. Paediatric Anaesthesia. 2018;28(2):174-8.

25. Fullington NM, Cauley RP, Potanos KM, O'Melia L, Zurakowski D, Bae Kim $\mathrm{H}$, et al. Immediate extubation after pediatric liver transplantation: a single-center experience. Liver Transplant. 2015;21(1):57-62.

26. Skurzak S, Stratta C, Schellino MM, Fop F, Andruetto P, Gallo M, et al. Extubation score in the operating room after liver transplantation. Acta Anaesth Scand. 2010;54(8):970-8.

27. Mandell MS, Lezotte D, Kam I, Zamudio S. Reduced use of intensive care after liver transplantation: influence of early extubation. Liver Transplant. 2002;8(8):676-81.

28. Blaszczyk B, Wronska B, Klukowski M, Flakiewicz E, Kolacz M, Jureczko L, et al. Factors Affecting Breathing Capacity and Early Tracheal Extubation After Liver Transplantation: Analysis of 506 Cases. Transplant P. 2016;48(5):1692-6.

29. Chae MS, Kim JW, Jung JY, Choi HJ, Chung HS, Park CS, et al. Analysis of pre- and intraoperative clinical for successful operating room extubation after living donor liver transplantation: a retrospective observational cohort study. BMC Anesthesiol. 2019;19(1):112.

30. Biancofiore G, Bindi ML, Romanelli AM, Boldrini A, Bisa M, Esposito M, et al. Fast track in liver transplantation: 5 years' experience. Eur J Anaesthesiol. 2005;22(8):584-90.

\section{Publisher's Note}

Springer Nature remains neutral with regard to jurisdictional claims in published maps and institutional affiliations.
Ready to submit your research? Choose BMC and benefit from:

- fast, convenient online submission

- thorough peer review by experienced researchers in your field

- rapid publication on acceptance

- support for research data, including large and complex data types

- gold Open Access which fosters wider collaboration and increased citations

- maximum visibility for your research: over $100 \mathrm{M}$ website views per year

At BMC, research is always in progress.

Learn more biomedcentral.com/submissions 